\title{
Classical and quantum stability in putative landscapes
}

\author{
Michael Dine \\ Santa Cruz Institute for Particle Physics and \\ Department of Physics, University of California at Santa Cruz, \\ Santa Cruz, CA 95064, U.S.A. \\ E-mail: dine@scipp.ucsc.edu
}

ABSTRACT: Landscape analyses often assume the existence of large numbers of fields, $N$, with all of the many couplings among these fields (subject to constraints such as local supersymmetry) selected independently and randomly from simple (say Gaussian) distributions. We point out that unitarity and perturbativity place significant constraints on behavior of couplings with $N$, eliminating otherwise puzzling results. In would-be flux compactifications of string theory, we point out that in order that there be large numbers of light fields, the compactification radii must scale as a positive power of $N$; scaling of couplings with $N$ may also be necessary for perturbativity. We show that in some simple string theory settings with large numbers of fields, for fixed $R$ and string coupling, one can bound certain sums of squares of couplings by order one numbers. This may argue for strong correlations, possibly calling into question the assumption of uncorrelated distributions. We consider implications of these considerations for classical and quantum stability of states without supersymmetry, with low energy supersymmetry arising from tuning of parameters, and with dynamical breaking of supersymmetry.

KEYwords: Supersymmetric Effective Theories, Supersymmetry Breaking

ARXiv EPrint: 1512.08125 


\section{Contents}

1 Introduction $\quad 1$

2 Multiple field landscape models 3

3 Scaling with $N \quad 4$

4 Lessons from string theory $\quad 6$

$\begin{array}{lll}4.1 & \text { Conditions on radii and couplings } & 6\end{array}$

4.2 Analogous questions in critical string theory 8

4.3 A string calculation 9

5 The approximately supersymmetric case $\quad \mathbf{1 2}$

$\begin{array}{lll}5.1 & \text { Roads to approximate supersymmetry in landscape models } & 12\end{array}$

$\begin{array}{lll}5.2 & \text { Classical stability with approximate supersymmetry } & 13\end{array}$

6 Landscape statistics at large $R \quad 14$

$\begin{array}{lll}7 & \text { Quantum stability } & 15\end{array}$

8 Conclusions $\quad 16$

\section{Introduction}

The notion that there is an underlying landscape leads to a picture in which there is an ensemble of theories with varying types and number of degrees of freedom at a high energy scale, and with lagrangian parameters at that scale picked at random from an underlying distribution. Flux vacua in various theories have served as models for understanding these questions [1]. If there are large numbers of quantized fluxes, each of which can take many values, the number of states can be exponentially large. Many analyses of this possibility have been conducted in string theory (e.g. IIB and F theory). In these theories, if the fluxes vanish, there are large numbers of moduli; moduli and fluxes are paired. Many of these fields may be stabilized and gain mass once fluxes are turned on, already at the level of the classical equations. The stabilization of these moduli is usually studied at the level of model effective field theories. In many cases $[2,3]$ the working assumption has been that one could describe the system with a locally supersymmetric lagrangian with a large number of fields, with Kahler potential and superpotential terms chosen from suitable (say Gaussian) distributions. Many of the results obtained from these studies appear robust, with a generic flavor. For example, distributions of supersymmetry breaking scales for 
small scales can be understood in terms of a low energy theory of a single chiral field, with parameters chosen from uniform distributions [4].

Another approach to modeling a landscape is to simply consider a field theory with a large number of fields, and make assumptions about the statistical distributions of lagrangian parameters $[5,6]$.We will refer to this as the large $N$ viewpoint. The relation noted above, between numbers of possible fluxes in string compactifications and moduli, suggests to many authors a connection between these two pictures. Much of our discussion in this paper will be in the context of systems with large numbers of fields. In such theories, it is relatively easy to formulate the problem of finding stationary points, and to consider issues of classical and quantum stability.

On the other hand, in a scenario in which most or all moduli are stabilized by a potential generated by fluxes, moduli masses typically scale as an inverse power of the compatification radius (for simplicity, we will think of a compatification with all compact dimensions of comparable size, $R$ ). In this case a large number of light pseudomoduli requires that $R$ be large; if this is not the case, it is not clear why the analysis in terms of many fields is sensible. We will argue that large $R$ is not likely to play a special role in determining the statistics of vacuum states, and that a priori one expects such states to be rare. In flux compactifications, then, reconciling large $N$ results, such as the fraction of stationary points exhibiting stability, with this expectation will provide consistency checks on assumptions about the underlying landscape.

In would-be vacua without supersymmetry, the questions of classical stability $[6,7]$ and quantum metastability $[8,9]$ have largely been explored in the large $\mathrm{N}$ framework (exceptions, for quantum tunneling, include [10]). These analyses make various assumptions about the low energy field content and statistics of the effective action of such states. An often unstated assumption must be made from the start: the couplings at these stationary points must be sufficiently weak that a semiclassical analysis is sensible; otherwise the notion of "stationary points" is not meaningful. Accepting this (and the conditions for and plausibility of this assumption will be one of the topics of this paper), naively, one might expect that among non-supersymmetric stationary points, a fraction of order $(1 / 2)^{N}$ would be stable or metastable. Various studies [6,7], however, suggest far more severe suppression, possibly as $e^{-c N^{2}}$ or more generally $e^{-a N^{p}}$, for some constant positive constants, $a, c$ and $p$. For approximately supersymmetric states, a high degree of metastability would seem almost assured [10], but the results of [7, 11] suggest that one should rethink this issue as well. As for quantum stability, among metastable states, without supersymmetry, one finds an exponential suppression of stability against tunneling, but the analyses make a number of assumptions, and it is not clear how general they are.

In this note we attempt to develop a more robust understanding of the question of stability. In section 2 , we discuss some general aspects of $N$-field models. In section 3 , under the assumption that couplings of fields are uncorrelated, we determine how couplings must scale with $N$ in order that there be a sensible perturbative expansion in the low energy effective field theory; more precisely that the low energy theory should not be in violent conflict with unitarity. In section 4 , we consider implications of these scalings for actual string models. We first note that in flux compactifications, the existence of large 
numbers of relatively light fields requires that the compactification radius grow as a power of the coupling, and discuss the power with particular frameworks. We argue that it is not plausible, with or without supersymmetry, that the preponderance of metastable states lie at large radius, and consider the connection of this issue with the question of stability for large $N$. We also note the possibility that, independent of the coupling and radii, sums of (squared) couplings do not grow with $N$. This question can be investigated in critical string theories, and in section 4.3, we carry through a simple computational test, demonstrating that indeed certain sums of squared couplings are of order one rather than order $N$. We argue that this is an indication of strong correlations among couplings.

In section 5, we consider states with approximate supersymmetry, with a particular focus on questions of stability and the possible role of large numbers of fields. We first review two models for supersymmetry in a landscape, one in which the hierarchy is achieved by tuning (of fluxes), another where it arises due to dynamical breaking of supersymmetry (in some form). We explain why one expects that stability, both classical and quantum, in a landscape is a feature of an order one fraction of stationary points. In the tuned case, we review the result of $[7,11]$, that stability is suppressed exponentially in the number of fields, $N$. We explain why this is surprising, and isolate the origin of the suppression. We argue that, if the requirements for unitarity (perturbativity) are satisfied, classical stability is typical. In section 5, we review ideas for different branches of the landscape, and discuss the implications of our observations. With small supersymmetry breaking, either through tuning or (especially for the case of) dynamical supersymmetry breaking, stability is not a significant constraint. In section 8, we conclude, with an overview and some final speculations on the possible role of supersymmetric and non-supersymmetric states in a landscape.

\section{Multiple field landscape models}

One picture for the emergence of a discretuum or landscape of states is that of Bousso and Polchinski [1], in which there are a large number of possible choices of fluxes, each defining a system with a unique (or small number of) (metastable) ground state(s). Another is that there are large numbers of fields, $N$, admitting a vast number of stationary points, some fraction of them stable [5]. In Calabi-Yau compactifications of string theories, there would appear to be a connection. In the case of IIB theories, for example, the number of possible $(2,1)$ forms is related to the number of complex structure moduli, which are massless in the absence of fluxes. So if one number is large, the other is large.

Theories with many fields provide a relatively simple setting in which to address questions of classical and quantum stability. As a simple starting point, which illustrates a number of the main issues, consider a theory with $N$ scalars, where the potential is simply a sum of terms

$$
V(\vec{\phi})=\sum_{i=1}^{N} f_{i}\left(\phi_{i}\right)
$$


Assume that the $f_{i}$ 's are all bounded below, and grow without bound at infinity. If a typical $f_{i}$ has $n$ minima and $n-1$ maxima, then the system has $(2 n-1)^{N}$ stationary points and $n^{N}$ minima. In other words, an exponentially small fraction of stationary points, $\left(2^{-N}\right.$ if $n$ is large), are classically stable. This picture may change drastically, as discussed in [6], once couplings of the different fields to each other are considered. If all couplings between fields are of order the couplings in the $f_{i}$ 's, an assumption we will refer to as coupling democracy, we might expect significantly more stationary points. At the same time, only an extremely tiny fraction would be true minima. This point was stressed in [6], The suppression of stability, these authors found, is of order $e^{-c N^{2}}$, for some constant $c$. If the couplings scale, instead, as some inverse power of $N$, or if only a few couplings are typically appreciable, then, without supersymmetry, the suppression of stability is only exponential.

Quantum stability can be addressed in a similar way. The authors of [8] considered tunneling in a theory with many fields. Assuming the existence of a minimum, they took the coefficients of the Taylor series expansion about that point to be independent random numbers. In numerical simulations, they found an exponential suppression of states with (logs of) lifetimes greater than some fixed value. These results were understood analytically in [9]. The analysis was particularly simple in the case where couplings between different fields were suppressed.

Ref. [12] considered the question of classical stability in the framework of locally supersymmetric lagrangians. These authors selected Kahler potentials and superpotentials from plausible random distributions. Without approximate supersymmetry, they found a suppression with $N$ behaving as $e^{-c N^{1.3}}$. We will discuss their results in the case of approximate supersymmetry shortly. One might argue that the use of a supersymmetric effective lagrangian is a strong assumption. Unless the scale of supersymmetry breaking is small, it is not clear why one should cut off the derivative expansion for the effective field theory at terms with two derivatives. This corresponds to cutting off the theory at terms with at most two powers of auxiliary fields. At least in cases where the supersymmetry breaking scale does turn out to be low, however, one can argue that the analysis is self-consistent. This suggests that the truth might lie in between the results of [6] and [12]. In either case, this is a very substantial suppression, suggesting that metastable states, at least without some approximate supersymmetry, might be quite rare. We will comment more on this issue shortly.

\section{Scaling with $N$}

In a theory with $N$ fields, the assumption that all couplings in an effective lagrangian are selected at random, from $N$-independent distributions, is not sensible as $N$ gets large. The theory will violate unitarity in perturbation theory. Radiative corrections will grow with powers of $N$ (in the supersymmetric case, we will discuss a potential violation of unitarity already at tree level shortly). Indeed this violent breakdown of perturbation theory will indicate a lack of even a qualitative understanding of the physics.

If we demand that couplings scale uniformly with $N$ so as to yield a sensible expansion, and assume, for simplicity, that the scale of non-renormalizable couplings is comparable 
to the ultraviolet cutoff (an assumption which we will assess later in the context of string theory), we can, by considering loop corrections, obtain scaling laws as a function of $N$. In particular, if we insist that successive terms in the perturbation expansion be as small or smaller by powers of $N$, and if there is just one characteristic mass scale, which we take, for simplicity, to be the Planck scale, $M_{p}$, we have

1. Without supersymmetry, terms in the potential of the form $\phi^{a}$ (we will confine our attention to $a>2$ ) should have couplings which scale with inverse powers of $N$. Writing these as

$$
\gamma_{i j k \ell \ldots} \phi^{i} \phi^{j} \phi^{k} \phi^{\ell} \ldots
$$

then the scaling

$$
\gamma^{(a)} \sim M_{p}^{-(a-4)} N^{-\frac{a}{2}}
$$

yields systematically small perturbative corrections. To see this, it is helpful to first examine some examples. Consider corrections to the two point function. If we consider $\left\langle\phi_{i} \phi_{i}\right\rangle$ ( $i$ not summed), then there is a correction, quadratic in the four point couplings, proportional to $N^{3}$. With the scaling above, the result behaves as $N^{-1}$. If we have different indices, $\left\langle\phi_{i} \phi_{j}\right\rangle$, we might expect the different contributions to add with random signs, and the result is further suppressed. Similarly, for the contribution from the six point, eight point functions. Corrections to the four point function are similarly suppressed.

One can possibly allow a slightly weaker suppression with $N$. The $a$-point functions which receive the largest corrections are those with some indices identical. It is possible that these are larger, by powers of $N$, than those with all indices different. In particular, the scaling for the couplings with different values for the indices,

$$
\gamma^{(a)} \sim M_{p}^{-(a-4)} N^{\frac{1-a}{2}} .
$$

while for those with indices equal in pairs:

$$
\gamma^{(a)} \sim M_{p}^{-(a-4)} N^{\frac{2-a}{2}} .
$$

appears sensible. We will not pursue this further, adopting the stronger scaling if necessary.

2. With supersymmetry, if the couplings in the superpotential with $a$ powers of chiral fields scale as

$$
\lambda^{(a)} \sim M_{p}^{3-a} N^{-(a-1) / 2}
$$

then perturbation theory appears under control. For example, the corrections to the Kahler potential scale as $N^{0}$. One can check that this coincides with a somewhat more severe suppression of scalar couplings in 3.2. The quartic coupling, for example, scales as $N^{-2}$. 
It should be stressed, again, that we are assuming here that there is just one relevant mass scale in the underlying theory, and that, apart from the scalings with $N$, there are no other parameterically very small parameters. Such parameters (small pure numbers or ratios of mass scales) could alter the considerations described here.

We can compare these behaviors with what one would expect if, in some basis for the fields, only the self couplings were of order one, while all of the others vanished (as some large power of $N$ ). We will refer to such couplings as sparse. For the case of real fields, for example, one could consider transforming the fields to another basis by a random $O(N)$ matrix, leading to couplings scaling as inverse powers of $N$. Then, for example, for the four point coupling,

$$
\gamma_{i j k l}=\sum_{m} O_{i m} O_{j m} O_{k m} O_{\ell m} \tilde{\gamma}_{m m m m}
$$

Typical entries in the orthogonal matrix $O$ would behave as $N^{-1 / 2}$, and assuming terms add with random signs, we would have

$$
\gamma_{i j k l} \propto N^{-3 / 2} .
$$

More generally, for a coupling involving $a$ real scalar fields,

$$
\gamma^{(a)} \propto N^{\frac{1-a}{2}} .
$$

This corresponds to our weaker condition, 3.3. For couplings with all indices paired, again we find a similar scaling as in equation (3.4).

\section{Lessons from string theory}

Modeling of landscapes has been heavily influenced by string theory; indeed, this is the only framework we have in which to make sense of higher dimensional theories, or even four dimensional theories with gravity. As we have stressed from the beginning, fluxes in string theory have provided both a key to how exponentially large numbers of states might arise, and to how large numbers of relatively light scalar fields might emerge. On the other hand, if the candidate light fields are the moduli which exist in the absence of fluxes, and these fields gain mass proportional to flux, it is not clear why, in general, these fields will be light compared to other string states. The standard (and most plausible) suggestion is that this will occur when compactification radii are large.

\subsection{Conditions on radii and couplings}

In the IIB theory, for example, complex structure moduli acquire potentials in the presence of fluxes. If typical fluxes are of order $M$, there is a $2 N \times 2 N$ mass matrix for the scalars, with typical entries

$$
\left(m_{c s m}^{2}\right)_{i j} \propto \frac{M^{2}}{R^{6}}
$$


(possibly with additional powers of $N$ ). If the matrix is very sparse, the eigenvalues are of order $M^{2}$, and we require

$$
R^{3} \gg M
$$

while if the entries are random and independent of each other (and $N$ ), we have the requirement:

$$
R^{3} \gg \sqrt{N} M
$$

In any case $R$ must be large, at least by powers of $M$, and quite possibly by powers of $N$ (this is certainly the case with the assumptions of [7]).

Requiring sufficiently weak coupling (perturbativity) is potentially an even stronger constraint. Couplings of complex structure moduli involving $n$ fields scale as

$$
\lambda^{(n)} \sim M^{2} R^{-4-n}
$$

So if $R$ is the source of suppression of couplings, it appears one may require even larger $R$ as a function of $N$. Alternatively, suppression may result from $g_{s}$ or from features of the string amplitudes themselves, as we will discuss shortly.

The problem of fixing the radial moduli, especially without supersymmetry or approximate supersymmetry, is not well understood. Assuming that such large $R$ is required, it is natural to ask how typical this might be. It is hard to make general statements about the non-supersymmetric case, but models for fixing radii with fluxes were put forward in the large extra dimension scenario of [13-17]. These involved playing off potentials due to fluxes with a bulk cosmological constant, curvature terms and possibly Casimir energies. The typical scalings were designed to allow very large extra dimensions and vanishing four dimensional cosmological constant. If terms of the first three types were of comparable importance, so as to allow cancellation of the c.c., then the typical radii satisfied $R \sim M^{1 / 3}$. This is not good enough to give rise to many light fields. Other scalings and tunings of parameters may allow more rapid growth of $R$ with $M$, but it seems that large radius with many light fields is not likely to be typical. In general, we might expect large $R$ to be associated with runaway behavior. An alternative approach is the exponentially large volume scenario of [18]. While perhaps not evidence for any particular phenomenon, the instability of typical vacua in large $\mathrm{N}$ models is consistent with the expectation that there should not be vast numbers of states at very large $R$. We will return to this issue in section 6 .

The KKLT model [19] provides a scenario, tied to approximate supersymmetry, in which a small fraction of states lie at large $R$. (As a model of moduli fixing in a landscape, it is necessary that the actual number of such states still be substantial). In this model, there is a large hierarchy between the masses of the majority of the moduli (complex structure moduli) and the lightest (Kahler) moduli, controlled not only by $R$ but by the parameter $W_{0}$ (the expectation value of the superpotential). Specifically, the radii are controlled by the Kahler modulus, $\rho \sim R^{4}$, which is itself of order:

$$
\rho=-\frac{1}{b} \log \left(W_{0}\right)
$$


where $b$ is a number of order one. So we require

$$
W_{0} \approx e^{-b \rho}
$$

In the KKLT scenario, this might be of order the fraction of states with $\mathrm{TeV}$ scale supersymmetry breaking, for example. In any case, this might be a substantial set of states where large $R$ is self-consistent. But as we will argue, large radius is not likely to be a driving feature in the population of approximately supersymmetric states, and not a critical issue in stability.

An alternative picture, also with approximate supersymmetry and exponentially large volume was put forward in ref. [18]. The framework was closely tied to string theory. A number of challenges for such a picture were discussed in [20], but if successfully met, this would fall in the general class of approximately supersymmetric theories we have discussed, and we would not expect any significant suppression of stability with the number of fields.

\subsection{Analogous questions in critical string theory}

We have seen that unitarity/perturbativity place restrictions on behavior of couplings with $N$. This might be enforced by large radius or weak string coupling. Alternatively, we have suggested a different possibility, that couplings might be "diagonal", in the sense that, for example, the set of substantial quartic couplings might be sparse. While it seems challenging to address this question in an actual flux landscape, we can consider similar issues in critical string theories with large numbers of fields.

We will consider supersymmetric theories, and suppose that we have a set of chiral fields, $\phi_{i}$, with Yukawa couplings (cubic superpotential couplings) among them. First, let's review the issue of unitarity/perturbativity in this context. Suppose $X$ is a particular chiral multiplet, and that it couples to a large number of chiral multiplets through couplings of the form $\gamma_{i} X^{2} \phi_{i}$ (this will be relevant to the question of stability in the supersymmetric case shortly. Consider the process $X+X \rightarrow X+X$ (where here $X$ denotes the scalar in the multiplet) at energies high compared to the masses of the $N \phi_{i}$ 's. The potential includes a term

$$
V(X)=\sum_{i=1}^{N}\left|\gamma_{i}\right|^{2}|X|^{4} .
$$

The cross section, then, behaves as

$$
\sigma(X+X \rightarrow X+X)=\frac{\left(\sum_{i=1}^{N}\left|\gamma_{i}\right|^{2}\right)^{2}}{s} .
$$

With the assumption that all of the $\gamma_{i}$ 's are chosen independently from the same distribution, the corresponding amplitude grows as $N^{2}$, and violates partial wave unitarity for large $N$ by a factor $N^{2}$; in other words, as one increases $N$, it would be necessary to take the string coupling small as a power of $N$. This would seem surprising. 


\subsection{A string calculation}

An example, which is relatively simple to analyze, is provided by compactification of the heterotic string on a Calabi-Yau space with large Euler number. While there is not a systematic large $N$ computation (as is also true for most landscape constructions) we can still reasonably ask whether large numbers appear in perturbative computations. This would imply, in a manner analogous to our landscape discussion, that a valid perturbation theory would require a large radius or very small $g$ (scaling as a power of $N$ ). Here we can focus on the cubic terms in the superpotential. Consider, for example, the wellstudied case of defined by the vanishing of a quintic polynomial in $C P^{4}$. There are 101 27 's (corresponding to 101 complex structure moduli). At the level of cubic superpotential terms, $27^{3}$, there are of order $10^{5}$ independent couplings. For special points in the moduli space, the actual number is much smaller, due to enhanced symmetries. For example, it is well known that there is a point with a $Z_{5}^{4} \times S_{5}$ symmetry [21]. At this point there are only a small number of non-vanishing couplings. If one considers, for example couplings of the type $(a, a, b)$, with fixed $a$, there is typically only one field $(b)$ which can combine to form an invariant, rather than 101. But at generic points in the moduli space, the symmetry is broken and one would expect all couplings to be non-vanishing.

On the other hand, there is a simple way to bound certain combinations of couplings, which we will see is relevant to this problem. This arises from considerations of two dimensional conformal field theory (analogous considerations may be applicable in the framework of four dimensional theories, e.g. [22]). In such theories, coupling constants can be extracted from correlation functions of two dimensional fields. Features of such correlation functions, even for rather complicated compactifications of the theory, can readily be extracted, particularly for large compactification radius, where the two-dimensional, "world-sheet" theory which governs the two dimensional dynamics, is weakly coupled.

Consider a four point function of vertex operators:

$$
G\left(z_{1}, z_{2}, z_{3}, z_{4}\right)_{a b}=\left\langle V_{a}\left(z_{1}\right) V_{b}\left(z_{2}\right) V_{a}\left(z_{3}\right) V_{b}\left(z_{4}\right)\right\rangle
$$

This correlator yields the $S$-matrix of the particles labeled by $a$ and $b$. If the leading term in the operator product expansion of $V_{a}$ and $V_{b}$ is:

$$
V_{a}\left(z_{1}\right) V_{b}\left(z_{2}\right)=c_{a b c} \frac{V_{c}}{\left|z_{1}-z_{2}\right|^{2}},
$$

the $c_{a b c}$ (up to normalization) are the three point couplings of the particles $a, b$ and $c$. If we take $z_{1} \rightarrow z_{2}, z_{3} \rightarrow z_{4}$,

$$
G\left(z_{1}, z_{2}, z_{3}, z_{4}\right)_{a b}=\frac{\sum_{c=1}^{N} c_{a b c}^{2}}{\left|z_{1}-z_{3}\right|^{2}\left|z_{1}-z_{2}\right|^{2}\left|z_{3}-z_{4}\right|^{4}}
$$

So if we can estimate or bound the four point function, we can bound the couplings of the fields $a, b$ to other fields. In particular, if we consider a Calabi-Yau space, for example, with very large $N$, growth of the sum with $N$ would require comparable growth of the correlation function. 
To illustrate the application of this type of analysis, consider the heterotic string compactified on a Calabi-Yau space. We consider this class of models because, as we will see, the computation of the correlators is particularly simple. We focus on cubic terms in the superpotential, i.e. terms of the type $27^{3}$ (we will comment on other string constructions in a future publication, as well as elaborating some technical aspects of the analysis below). Let's consider a Green's function which receives a contribution from the cubic superpotential terms, involving two fermions and two bosons. For definiteness and because of its simplicity, we work in the fermionic formulation for the gauge degrees of freedom of the string theory, and in the R-NS formulation for the right moving fermions. Then the spatial coordinates can be grouped as $y^{i}, y^{\bar{i}}, x^{\mu}$, where the $i, \bar{i}$ are complex indices for the six dimensional Kahler manifold, and $\mu$ are four dimensional Minkowski indices. The left moving fermions are $\lambda^{i}, \lambda^{\bar{i}}, \lambda^{a}$, where the $a$ 's are $O(10)$ indices. Space-time spinor operators can be taken as $S_{\alpha}^{0}, S_{\alpha}^{i}, S_{\alpha}^{\overline{0}}, S_{\alpha}^{\bar{i}}$, where $\alpha$ are four dimensional spinor indices. The 0 and $\overline{0}$ indices correspond to the covariantly constant spinor. At large radius, the theory is nearly free, and these operators reduce to their free field forms. Bosonizing the right moving fermions,

$$
\psi^{i}=e^{i \phi_{i}}
$$

for the fermions with indices in the compact space, whereas for the $\psi$ 's with Minkowski indices

$$
\left(\psi_{1}+i \psi_{2}\right)=e^{i \xi_{1}} \quad\left(\psi_{3}+i \psi_{4}\right)=e^{i \xi_{2}}
$$

Then

$$
S^{0}=c e^{\frac{i}{2}\left(\phi_{1}+\phi_{2}+\phi_{3}\right)} e^{\frac{i}{2}\left( \pm \xi_{1} \pm \xi_{2}\right)} \quad S^{\overline{0}}=c e^{-\frac{i}{2}\left(\phi_{1}+\phi_{2}+\phi_{3}\right)} e^{\frac{i}{2}\left( \pm \xi_{1} \pm \xi_{2}\right)}
$$

where in the first case there are an even number of plus signs, the second an odd number. The $S_{i}$ 's are given by

$$
S_{\alpha}^{i}=c e^{\frac{i}{2}\left(\phi_{1}+\phi_{2}-\phi_{3}\right)} e^{\frac{i}{2}\left( \pm \chi_{1} \pm \chi_{2}\right)} \quad S_{\alpha}^{\bar{i}}=c e^{-\frac{i}{2}\left(\phi_{1}+\phi_{2}-\phi_{3}\right)} e^{\frac{i}{2}\left( \pm \xi_{1} \pm \xi_{2}\right)}
$$

(this is $S^{3}, S^{\overline{3}}$; other values of the index are obtained by changing the placement of the minus sign in the first exponent). Decomposing the 27 into representations of $O(10) \times \mathrm{U}(1)$,

$$
27=16_{-1 / 2}+10_{1}+1_{-2}
$$

the boson vertex operators for particles in the 1 can be taken to be:

$$
V_{B}=\lambda^{\bar{i}} \lambda^{\bar{j}} \psi^{k} \chi_{k i j}^{(\alpha)}
$$

and its complex conjugate. $\chi_{k \bar{i} j}^{(\alpha)}$ is a harmonic $(2,1)$ form. It is related to the corresponding fluctuation in the metric, $\delta g_{i j}^{\alpha}$ through

$$
\delta g_{i j}^{(\alpha)}=\chi_{\bar{k} \bar{l}}^{\bar{m}(\alpha)} \Omega^{\bar{k} \bar{l} \bar{n}} g_{j \bar{m}} g_{i \bar{n}} .
$$


where $\Omega_{i j k}$ is the covariantly constant three form. We will write the fermion vertex operator, for particles in the 10 representation, as:

$$
V_{F}=\epsilon_{\alpha}^{i} \lambda^{a} \lambda^{i} S_{\alpha}^{j} \delta g_{i j}^{(\alpha)}
$$

(it is also necessary to include suitable superconformal ghost fields in these vertex operators).

We consider the scattering of one fermion corresponding to the $(\alpha)^{\prime}$ 'th $(2,1)$ form with a scalar corresponding to the $\beta$ 'th $(2,1)$ form, to produce the same fermion and boson (elastic scattering). This arises through the cubic terms in the superpotential,

$$
\gamma_{\alpha \beta \gamma} \Phi^{\alpha} \Phi^{\beta} \Phi^{\gamma}
$$

In a sigma model description, at large $R$, the singular parts of the Green's function are readily evaluated, as the correlation functions of the $\phi_{i}$ 's and $\chi_{i}$ 's (as well as the ghosts, which we have suppressed) are those of free fields. The usual $\delta$ function for energy and momentum conservation in the compactified directions is replaced by an integral over the compact dimensions. The coefficient of

$$
\frac{1}{\left|z_{1}-z_{3}\right|^{4}\left|z_{1}-z_{2}\right|^{2}\left|z_{3}-z_{4}\right|^{2}}
$$

is

$$
\mathcal{A}=\int d^{6} y \delta g_{i j}^{(\alpha)} \delta g_{k l}^{(\beta)}\left(\delta g^{*(\alpha) i j} \delta g^{*(\beta) k l}-\delta g^{*(\alpha) i k} \delta g^{*(\beta) j l}\right)
$$

The $\delta g$ 's are normalized to unity. As a result, the integral does not show growth with the number of complex structure moduli ( $h_{2,1}$ of the manifold) unless the $\delta g$ 's are singular. On the other hand

$$
\mathcal{A}=\sum_{k}\left|\gamma_{i j k}\right|^{2}
$$

which is now of order 1 rather than of order $N$.

This argument might fail if $\mathcal{A}$ were enhanced with $N$. This in turn requires that the integral above be singular. Taking the $\delta g$ 's to be normalized, we can bound $\mathcal{A}$ by $\max |\delta g|^{2}$. So we would require that the maximum be of order some power of $N$. At the highly symmetric point in the moduli space studied in [21], there is no such enhancement, and it would be surprising if it arose as we turned out various complex structure moduli.

Another argument for suppression comes from thinking about the mirror manifold. ${ }^{1}$ For the mirror, the Yukawa's we compute here are just intersection numbers. These are expected to be sparse. Applications of this type of analysis to other string theories and types of compactifications, as well as further investigations of possible loopholes, will be considered in a future publication.

\footnotetext{
${ }^{1}$ I thank S. Kachru for help in refining this argument.
} 


\section{The approximately supersymmetric case}

For many years, supersymmetry has been viewed as a promising framework in which to resolve the hierarchy problem. In a landscape framework, it is not clear whether supersymmetry is particular important for questions of naturalness; it is simply possible that there are overwhelmingly more non-supersymmetric than supersymmetric states [23, 24]. Indeed, one might think that supersymmetry is exceptional among possible states in a landscape. The issue of stability, however, suggests a special role for supersymmetry. At least naively, one would expect that approximate supersymmetry would greatly increase the chances for stability, as exact supersymmetry guarantees exact stability. So given the possibly extreme suppression of stability we have seen without supersymmetry, it is conceivable that supersymmetric states are more common than non-supersymmetric states.

\subsection{Roads to approximate supersymmetry in landscape models}

Approximate supersymmetry has been modeled in landscapes in various ways. Douglas and Denef [25], starting with an underlying supergravity theory, and making assumptions about distributions of parameters, argued that approximate supersymmetry was highly suppressed. This was shown to follow [4] from the assumption that such theories are described, at low energies, by a single light chiral field, $Z$, with superpotential parameters uniformly distributed as complex numbers, and Kahler potential parameters as real numbers. This suppression, as the supersymmetry breaking scale, $F$, to the sixth power, was seen as an argument that such theories do not resolve the hierarchy problem in a natural way. Indeed, in this branch of the landscape, low energy supersymmetry, when it occurs, is an accident, arising from tuning fluxes to obtain a low energy theory with approximate supersymmetry. We will refer to models of this type as models with tuned supersymmetry.

Within theories with $N$ fields, on such a branch, one field is by assumption far lighter than the others. The "price" of this tuning is part of the $F^{6}$ suppression. With this assumption, it would seem surprising that there would be extreme suppression beyond that of [25] from the requirement of stability. Indeed, as there is no requirement of an additional light chiral field, one would expect that all of the other moduli are typically heavy. For example, if almost all of the moduli have masses suppressed by powers of $R$, while the lightest modulus is far lighter, then we would not expect a dramatic, qualitative change as the radius becomes of order one. Indeed, we will argue in the next subsection that there is likely, at most, an order one suppression

A more plausible setting for supersymmetry arises if the breaking is dynamical [26, 27]. In this case, while very low energy breaking of supersymmetry is not necessarily favored, it is typically not disfavored. Such theories exhibit an exponential gap between the scales of supersymmetry breaking and other states. Certainly the presence of $N$ relatively heavy "moduli" should not have a strong bearing on questions of stability. We will understand these points in the next section. 


\subsection{Classical stability with approximate supersymmetry}

Refs. [7, 11], studied theories with approximate supersymmetry (in the sense of tuned supersymmetry, defined above) and $N$ massive fields. Here "massive" means heavy compared to any scale of supersymmetry breaking, but light compared to the string (or other fundamental) scale. They argued that one expects an exponential suppression of classical stability, i.e. only an order $e^{-c N}$ fraction of states are classically stable.

At first sight, this result is puzzling. In these cases, one would expect that there is a scale with approximate supersymmetry with only one chiral field. There would then be only a small number of important parameters in the effective action. While there would be some constraints on these parameters, it is surprising that the stability of a few light scalar fields is exponentially sensitivity to the number of heavy fields (indeed, any significant suppression with $N$ ).

Calling the Goldstino supermultiplet $X$, with the scalings with $N$ we have described in eq. (3.5), we can take its superpotential to be $f X$, and its Kahler potential $X^{\dagger} X$, up to terms suppressed by powers of $N$. Requiring (nearly) vanishing c.c., would yield exactly the Polonyi model, for which the scalar fields have positive mass-squared. Negative masssquared would seem not only unlikely but impossible.

It is easy, however, to identify where the sensitivity to the number of heavy fields arises. Assume that, in addition to $X$, there are $N$ heavy fields, $\Phi_{i}$. Suppose the superpotential for $X$ and $\Phi_{i}$ is:

$$
W=X f+\sum_{i=1}^{N}\left(\frac{1}{2} m_{i}^{2} \Phi_{i} \Phi_{i}+\gamma_{i} X^{2} \Phi_{i}\right) .
$$

Integrating out the massive fields generates a correction to the Kahler potential:

$$
\delta K=\sum_{i=1}^{N} \frac{\left|\gamma_{i}^{2} \| X^{\dagger} X\right|^{2}}{m_{i}^{2}} .
$$

This leads to a correction to the mass of $X$. One can calculate this easily by looking directly at the potential and integrating out the $\Phi_{i}$ fields by solving for the minimum of their potential as a function of $X$. The result is:

$$
m_{X}^{2}=-\sum_{i=1}^{N} \frac{\left|\gamma_{i}\right|^{2}}{4 m_{i}^{2}}
$$

in agreement with formulas in [12].

It is important to understand in this expression how the various couplings scale with $N$. If, for example, as suggested in $[7,11]$, the $\gamma$ 's scale as $1 / \sqrt{N}$, given that there are $N$ positive terms in the sum, whether the $X$ mass is typically tachyonic depends on how $1 / m_{i}^{2}$ behaves. The mass matrix is of the form $m^{\dagger} m$ (a Wishart matrix, see, e.g. [7]). These authors assumed that the the entries in $m$ are of order $1 / \sqrt{N}$ As a result, the typical masses are of order 1 , but there are frequent fluctuations to low eigenvalues, and it is rare that the sum is small; indeed, a numerical study indicates that the sum is typically of order $N$. With these assumptions, only exponentially rare downward fluctuations will lead to stability. 
We have advocated a different scaling above, one which is compatible with perturbativity (and also with diagonal couplings). With these scalings, there is an additional $1 / N$ (the scaling of masses is the same, but the couplings at the vertices scale as $1 / N$ ). As a result, the sum of terms is typically of order one, i.e. comparable to the leading Polonyi term. (Here, the couplings give $1 / N^{2}$, the inverse mass squared factor gives a factor of order $N$, and the overall number of terms gives an additional $N$ ). So these affects don't grow with $N$. As a result, the chances of stability are of order one. Note that this extra contribution to the Kahler potential is soft, and so consistent with our scalings.

It should be noted that there might be additional suppression of masses. In the string context, this could arise due to large $R$, as we have discussed. In this case, however, there is additional, compensating dependence of couplings with $R$, and as a result one does not expect further difficulties with stability.

This does not change the fact that tuned supersymmetry is expected to be rare in a landscape. On the other hand, heavy fields do not pose any issue for dynamical supersymmetry breaking. In such theories, the mass of partners of the Goldstino (if such can be identified) are typically of order the scale of supersymmetry breaking, rather than the gravitino mass. So the contributions to the Kahler potential from integrating out very massive fields are totally inconsequential.

\section{$6 \quad$ Landscape statistics at large $R$}

We can ask whether the various speculations about stability are compatible with the picture of large numbers of fields arising at large $R$ in compactified string theories. The presumption of an exponentially large number of states at large $R$, we have already argued, is not terribly plausible. Another way to see the puzzle this raises is to consider how the distribution of states might behave with $R$. If for large $R$, there is an exponentially large number of states with some parameter $N$, do the majority of these simply disappear along with the parameter $N$ - as $R$ decreases? This would seem particularly troubling in the supersymmetric case. If not, what would play the role of the parameter $N$ at small $R$ ?

The high level of instability in the non-supersymmetric case would seem to avoid this paradox. The exponential suppression could be take to indicate that there is typically at most of order one metastable state per flux choice. Assuming this is the case (and that the number is not significantly smaller than one), the question becomes: what is the rate for tunneling between states with different flux. In other words, quantum stability becomes the critical issue.

In the case of tuned supersymmetry, one does not expect particularly strong dependence on $R$ in the counting of states, and this is compatible with our arguments that there is no $N$ dependent suppression of stability. Such a picture, indeed, avoids any puzzles about discontinuous changes in the population of states as $R$ is varied; the same level of tuning is required in any case. With dynamical supersymmetry breaking, this issue does not arise. 


\section{Quantum stability}

In the event that a state is classically stable, it is necessary to ask about quantum stability. This question takes on particular significance in a landscape context. In the flux vaccum picture, any would-be nearly Minkowski vacuum will be surrounded by vast numbers of states with negative cosmological constant. With large numbers of fields, there will be many possible tunneling trajectories. Every one of these must be significantly suppressed.

This problem has been discussed elsewhere, but the observations of [7] and this paper permit one to make further statements about stability against tunneling. In [8], models with large numbers of fields were studied (without supersymmetry). Starting with a wouldbe classical ground state, the authors Taylor-expanded the potential, and assumed that the coefficients of the expansion were uniformly distributed random variables. In [9], the results of [8] were understood in a simple way: roughly speaking, the most probable tunneling trajectory is a straight line in field space in the direction of the lightest field. Suppressing tunneling then requires that all masses be larger than some minimum value. More precisely, the ratio of $\frac{\mu^{2}}{\gamma^{2}}$, where $\gamma$ is the cubic coupling in the direction of the field of mass $\mu$, for every choice of field, must be larger than some minimum value. With the assumption of uniform distribution of couplings, with the intervals considered in [8], this leads to exponentially small probability for bounce actions all greater than some minimum, $B_{0}$.

This analysis must be rethought in light of these observations. First, the assumption that all couplings are chosen from the same, $N$-independent random distributions, runs afoul of unitarity and perturbativity. If we assume, instead, $N$-dependent distributions, with couplings falling as powers of $N$, then qualitatively different results than those of $[8,9]$ may emerge. In particular, if the cubic couplings fall off more rapidly than $\mu^{2}$ with $N$, then bounce actions may be enhanced and tunneling suppressed. Indeed, the scalings above would suggest that the typical bounce action grows rapidly with $N$. Alternatively, if couplings and masses are diagonal in the same basis, the analysis of [9] implies significant further suppression of the tunneling. Indeed, it would seem surprising if classical stability were highly suppressed, but most classically stable vacua were highly metastable. Other than arguing that this is counterintuitive, however, we don't have a sharp argument for this type of alignment.

In flux landscapes, for $R \sim 1$, with an order one number of states per choice of flux, without supersymmetry, ref. [10] argued that tunneling rates were fast, with bounce actions behaving as inverse powers of flux.

With approximate supersymmetry, as stressed in [10], the situation is different. In field directions with curvature much greater than $m_{3 / 2}$, tunneling is highly suppressed, either vanishing or scaling as

$$
\Gamma \propto e^{-M_{p}^{2} / m_{3 / 2}^{2}}
$$

Assuming that there are only a small number of fields with mass of order $m_{3 / 2}$ only a few tunneling amplitudes need to be suppressed ((roughly corresponding to tunneling in directions connected with the goldstino multiplet), so one does not expect to pay an exponential price. These statements hold not only for dynamical supersymmetry breaking, 
where classical stability is not a severe constraint, but also to the case where low energy supersymmetry is the result of tuning of parameters (fluxes).

\section{Conclusions}

The existence of a landscape remains a subject of conjecture, based mainly on considerations of models of multiple fields and on the many candidate choices of flux in string theories. In this context, questions of stability, classical and quantum, can contribute both to assessment of the plausibility of the basic picture, and to understanding what features a putative landscape might exhibit. In the second category, from the beginning there has been a question: does supersymmetry play any role? Underlying this question is a view that supersymmetric states might be exceptional, a view which finds support in the work of Douglas and Denef [25], indicating that small supersymmetry breaking is rare.

The considerations of stability which we have reviewed and extended here provide insight into both classes of questions. The work of [7] (and earlier [6]) indicates that, without supersymmetry, classical stability might be extraordinarily rare. Within flux vacua, this might mean that for a given choice of flux, there is at most one stable state (with runaway likely being typical). We have indicated that some of the assumptions which go into this analysis might not be correct; critical string theories give some evidence that couplings may be highly correlated, and perturbativity requires scalings of couplings similar to what one would expect were they diagonal in some basis. At the same time, we have argued that large numbers of fields (moduli) in string theory compactifications with fluxes require very large radius, and that this is likely to be very rare. But our remarks on this question are only conjectural.

Without supersymmetry, quantum stability potentially provides further constraints, but these are also dependent on assumptions about distributions of couplings. If we require perturbativity but assume couplings are uncorrelated, the additional suppression could be quite mild; but further exponential suppression is also a possibility.

Supersymmetry (i.e. supersymmetry broken at scales well below the fundamental scale) does appear to occupy a priviedged place in any would-be landscape. General principles suggest that stability should be typical of states found in any sort of analysis. We have distinguished tuned supersymmetry breaking and dynamical breaking. In the former case, we have understood a possible source of instability noted in [7], and that this is unlikely to yield further appreciable suppression. We have also noted that for dynamical supersymmetry breaking, it is irrelevant.

We have discussed the question of large numbers of fields within flux landscapes. We have noted that such a picture is only valid when the compactification radius is large, scaling with a power of the flux. The power itself depends on assumptions about the nature of the underlying effective action. From the start, the existence of exponentially larger numbers of states at large radius relative to those at smaller scale is not terribly plausible. We have put forward a consistency condition that the spectrum of stable states should not depend critically on $R$. For the non-supersymmetric states, this is consistent 
with a vast suppression. For the supersymmetric case, our discussion suggests that large $R$ is not special.

If there is of order one non-supersymmetric state, classically, for typical choices of fluxes, absent supersymmetry there remains the question of quantum stability. Arguments that instability is typical, in this case, were put forward in [10].

There are a few principled things one can say about the case of approximate supersymmetry.

1. With zero cosmological constant and exact supersymmetry states are automatically stable.

2. With approximate supersymmetry and small cosmological constant, there are only a small number of relevant light fields (i.e. we do not expect scaling with $N$ ), so one does not expect exponential suppression of either classical or quantum stability with $N$.

3. Supersymmetry and approximate supersymmetry also guarantee stability in cases where, for a fixed choice of flux, there are only small numbers of states.

All of this suggests that supersymmetry, at a scale significantly below the fundamental scale, is likely to be important in a landscape. Stability does not, by itself, argue for TeV scale supersymmetry.

\section{Acknowledgments}

We thank Raphael Bousso, Shamit Kachru, Liam McAlister, Yasnori Nomura, Steve Shenker and Sonya Paban for sharing their insights into these questions. This work was supported by the U.S. Department of Energy grant number DE-SC0010107.

Open Access. This article is distributed under the terms of the Creative Commons Attribution License (CC-BY 4.0), which permits any use, distribution and reproduction in any medium, provided the original author(s) and source are credited.

\section{References}

[1] R. Bousso and J. Polchinski, Quantization of four form fluxes and dynamical neutralization of the cosmological constant, JHEP 06 (2000) 006 [hep-th/0004134] [INSPIRE].

[2] F. Denef and M.R. Douglas, Distributions of flux vacua, JHEP 05 (2004) 072 [hep-th/0404116] [INSPIRE].

[3] F. Denef and M.R. Douglas, Distributions of nonsupersymmetric flux vacua, JHEP 03 (2005) 061 [hep-th/0411183] [INSPIRE].

[4] M. Dine and Z. Sun, R symmetries in the landscape, JHEP 01 (2006) 129 [hep-th/0506246] [INSPIRE].

[5] N. Arkani-Hamed, S. Dimopoulos and S. Kachru, Predictive landscapes and new physics at a TeV, hep-th/0501082 [INSPIRE]. 
[6] A. Aazami and R. Easther, Cosmology from random multifield potentials, JCAP 03 (2006) 013 [hep-th/0512050] [INSPIRE].

[7] D. Marsh, L. McAllister and T. Wrase, The Wasteland of Random Supergravities, JHEP 03 (2012) 102 [arXiv:1112.3034] [INSPIRE].

[8] B. Greene, D. Kagan, A. Masoumi, D. Mehta, E.J. Weinberg and X. Xiao, Tumbling through a landscape: Evidence of instabilities in high-dimensional moduli spaces, Phys. Rev. D 88 (2013) 026005 [arXiv: 1303.4428] [INSPIRE].

[9] M. Dine and S. Paban, Tunneling in Theories with Many Fields, JHEP 10 (2015) 088 [arXiv: 1506.06428] [INSPIRE].

[10] M. Dine, G. Festuccia and A. Morisse, The Fate of Nearly Supersymmetric Vacua, JHEP 09 (2009) 013 [arXiv:0901.1169] [INSPIRE].

[11] T.C. Bachlechner, On Gaussian Random Supergravity, JHEP 04 (2014) 054 [arXiv:1401.6187] [INSPIRE].

[12] D. Marsh, L. McAllister and T. Wrase, The Wasteland of Random Supergravities, JHEP 03 (2012) 102 [arXiv:1112.3034] [INSPIRE].

[13] N. Arkani-Hamed, S. Dimopoulos and G.R. Dvali, Phenomenology, astrophysics and cosmology of theories with submillimeter dimensions and TeV scale quantum gravity, Phys. Rev. D 59 (1999) 086004 [hep-ph/9807344] [INSPIRE].

[14] R. Sundrum, Effective field theory for a three-brane universe, Phys. Rev. D 59 (1999) 085009 [hep-ph/9805471] [INSPIRE].

[15] R. Sundrum, Compactification for a three-brane universe, Phys. Rev. D 59 (1999) 085010 [hep-ph/9807348] [INSPIRE].

[16] N. Arkani-Hamed, S. Dimopoulos and J. March-Russell, Stabilization of submillimeter dimensions: The New guise of the hierarchy problem, Phys. Rev. D 63 (2001) 064020 [hep-th/9809124] [INSPIRE].

[17] T. Banks, M. Dine and A.E. Nelson, Constraints on theories with large extra dimensions, JHEP 06 (1999) 014 [hep-th/9903019] [INSPIRE].

[18] J.P. Conlon, F. Quevedo and K. Suruliz, Large-volume flux compactifications: Moduli spectrum and D3/D7 soft supersymmetry breaking, JHEP 08 (2005) 007 [hep-th/0505076] [INSPIRE].

[19] S. Kachru, R. Kallosh, A.D. Linde and S.P. Trivedi, de Sitter vacua in string theory, Phys. Rev. D 68 (2003) 046005 [hep-th/0301240] [INSPIRE].

[20] M. Dine, G. Festuccia, J. Kehayias and W. Wu, Axions in the Landscape and String Theory, JHEP 01 (2011) 012 [arXiv: 1010.4803] [INSPIRE].

[21] M.B. Green, J.H. Schwarz and E. Witten, Superstring Theory. Vol. 2: Loop Amplitudes, Anomalies and Phenomenology, Cambridge University Press, Cambridge U.K. (1988).

[22] F. Caracciolo and V.S. Rychkov, Rigorous Limits on the Interaction Strength in Quantum Field Theory, Phys. Rev. D 81 (2010) 085037 [arXiv:0912.2726] [INSPIRE].

[23] M.R. Douglas, The String landscape and low energy supersymmetry, arXiv:1204.6626.

[24] L. Susskind, Supersymmetry breaking in the anthropic landscape, hep-th/0405189 [INSPIRE]. 
[25] F. Denef and M.R. Douglas, Distributions of nonsupersymmetric flux vacua, JHEP 03 (2005) 061 [hep-th/0411183] [INSPIRE].

[26] M. Dine, D. O'Neil and Z. Sun, Branches of the landscape, JHEP 07 (2005) 014 [hep-th/0501214] [INSPIRE].

[27] M. Dine, E. Gorbatov and S.D. Thomas, Low energy supersymmetry from the landscape, JHEP 08 (2008) 098 [hep-th/0407043] [INSPIRE]. 